\title{
Educación y política en filosofía con niños y niñas. Reflexiones a partir de El maestro ignorante de Rancière*
}

Julián Macías*

Recibido: 8 de Agosto de 2014 - Revisado: 26 de octubre de 2014 -

Aprobado: 12 de diciembre de 2014

El presente trabajo gira en torno al objetivo, en cuanto a su politicidad, de la práctica de filosofía con niños y niñas. Por un lado, buscaremos poner de manifiesto las tensiones y paradojas que implica pensar la filosofía como una praxis educativa y política, y, por otro, ensayaremos una posible respuesta que permita tanto habitar esas tensiones y paradojas, como generar una transformación en las mismas prácticas educativas. Para ello, el trabajo será dividido en tres partes. Primero, el análisis de la propuesta Philosophy for Children de Lipman, enfatizando el concepto de comunidad de indagación y el rol que se le asigna al docente en dicho programa. En la segunda parte, bajo la hipótesis de que, si bien dicha propuesta se presenta a sí misma como innovadora, subyacen ciertos elementos que permitirían poner en cuestionamiento esa calificación, por lo que intentaremos mostrar las semejanzas con ciertos elementos de lo que podría denominarse "educación tradicional". Por último, examinaremos algunos elementos del planteamiento ranceriano en el Maestro ignorante respecto a la relación docente-alumno. En la misma lógica de los apartados anteriores, analizaremos si efectivamente se plantea una propuesta diferente y hasta qué punto es posible pensar una diversa relación.

Palabras clave: Filosofía para niños, Rancière, Lipman, educación política, comunidad de indagación

\footnotetext{
* $\quad$ Artículo de reflexión, que amplía y desarrolla cuestiones presentadas en un trabajo más breve, leído en el XXI Jornadas sobre la Enseñanza de la Filosofía-Coloquio Internacional (abril de 2014), y retoma ideas producidas en el marco del seminario de grado "El lugar del otro en la educación. Tensiones éticas en los procesos de enseñanza y aprendizaje", dictado por el Dr. Berisso en la Universidad de Buenos Aires.

** Profesor de Educación Media y Superior en Filosofía, Facultado de Filosofía y Letras, Universidad de Buenos Aires. Ayudante de la cátedra de Historia de la Filosofía Antigua. Dirección postal: Morello 3349, Buenos Aires, Argentina (CP: 1651). Correo electrónico: julianmacias85@gmail.com.
} 


\section{Education and politics in philosophy with children. Reflections based on The Ignorant Schoolmaster from Rancière*}

Julián Macías*

In the present work we will reflect on the goal, in terms of its political aspect, of practicing philosophy with children. On the one hand, we intend to show the tensions and the paradoxes that are implied when thinking philosophy as an educational and political praxis. On the other hand, we will try out a possible answer that allows us not only to inhabit those tensions and paradoxes, but also that enables a transformation of educational practices. In order to achieve the above, the paper is organized in three sections. In the first one, we will analyze Lipman's Philosophy for Children programme, emphasizing on the concept of community of Inquiry and the teaching role assigned by this proposal. In the second section - under the hypothesis that, although Philosophy for children is acknowledged as an innovative programme, there are some elements that allow us to question that qualification -we will try to show some similarities between Lipman's proposal and what is usually known as "traditional education". Finally, we will analyze some elements from Ranciere's The Ignorant Schoolmaster, in order to examine the student-teacher relationship proposed by the author. We will examine up close if such proposal is different from others, whether it is possible to think a different relationship between students and teachers.

Keywords: Philosophy for children, Rancière, Lipman, political education, community of inquiry

\footnotetext{
* Reflection article that takes as its starting point the work of the author and translator of the works authorized Lipman for Colombia and Philosophy for research

** PhD in philosophy at the Javeriana University, an institution where he currently serves as Dean of the Faculty of Philosophy. Address: Carrera 7 40-62, Bogota, Colombia. Email: diegoantpineda @ yahoo.com, diegopi@javeriana.edu.co.
} 


\section{Education et politique dans philosophie avec filles et garçons. Réflexions à partir du Maître ignorant de Rancière*}

Julián Macías*

\section{Résumé}

Ce travail tourne autour de l'objectif, dans sa nature politique, de la pratique de la philosophie avec les enfants. D'une part, nous cherchons à mettre en évidence les tensions et les paradoxes qui implique penser la philosophie comme une pratique éducative et politique, et d'autre part, on tentera d'obtenir une possible réponse qui permet à la fois d'aborder ces tensions et paradoxes, ainsi que générer une transformation au sein des pratiques d'éducation. Pour cela, le travail sera divisé en trois parties. Tout d'abord, l'analyse de la proposition Philosophy for Children de Lipman, en insistant sur la notion de communauté de recherche et le rôle attribué à l'enseignant dans le programme. Dans la deuxième partie, sous l'hypothèse que, malgré que la proposition se présente elle-même comme innovatrice, il y a des éléments qui permettraient de remettre en question cette qualification, c'est pourquoi on tentera de démontrer les similitudes avec certains éléments de ce que l'on pourrait appeler "l'éducation traditionnelle". Enfin, nous examinons certains éléments de l'approche " rancièrien » dans le Maître ignorant en ce qui concerne la relation enseignant-élève. Dans la même logique des sections précédentes, on fera une analyse de manière à savoir si effectivement il y a une proposition innovante et dans quelle mesure il est possible de penser une relation différente.

Mots clés: Philosophie pour enfants, Rancière, Lipman, éducation politique, communauté de recherche

L'article de réflexion qui prend comme point de départ le travail de l'auteur et traducteur des oeuvres autorisées Lipman pour la Colombie et la philosophie pour la recherche

** Doctorat en philosophie à l'Université Javeriana, une institution où il est actuellement doyen de la Faculté de Philosophie. Adresse: Carrera 7 40-62, Bogota, Colombie. Email: diegoantpineda @ yahoo.com, diegopi@javeriana.edu.co. 
Si los niños consiguen que se oigan sus protestas en una Maternal, o incluso simplemente sus preguntas, esto sería suficiente para producir una explosión en el conjunto del sistema de la enseñanza: verdaderamente, este sistema en el que vivimos no puede soportar nada: de ahísu fragilidad radical en cada punto, al mismo tiempo que su fuerza de represión global. A mi juicio usted ha sido el primero en enseñarnos algo fundamental, a la vez en sus libros y en un terreno práctico: la indignidad de hablar por los otros. ${ }^{1}$

Si bien la tensión entre la filosofía y la posibilidad de su enseñanza ha sido objeto de numerosas reflexiones filosóficas, probablemente haya sido Jacques Derrida quien captó en toda su dimensión la relación paradojal entre enseñanza y filosofía. Con cierta familiaridad a la afirmación kantiana "no se aprende filosofía, sino que se aprende a filosofar" (Crítica de la razón pura, II, 401), Derrida sostuvo que "la esencia de la filosofía excluye la enseñanza, [mientras que] la esencia del filosofar la exige" $(1990,368)$, enfatizando así el hecho de que el continuo devenir filosófico escapa a la quietud de cualquier método, herramienta, recurso o planificación.

Esta reflexión derridiana, lejos de clausurar los debates en torno enseñanza de la filosofía, se presenta como una oportunidad para pensar y repensar, no sólo el modo de hacer filosofía con niños/as, sino también la posibilidad misma de esta práctica. ¿Qué implica enseñar filosofía? ¿Cuál es su funcionalidad política? ¿Es posible escindirla de la política?

Ante estos interrogantes, en el presente trabajo intentaremos plantear cuál debería ser, a nuestro criterio, el objetivo, en cuanto a su politicidad, de la práctica de filosofía con niños y niñas. Por un lado, buscaremos poner de manifiesto las tensiones y paradojas que implica pensar a la filosofía como una praxis educativa y política, y, por otro, ensayaremos una posible respuesta que permita tanto habitar esas tensiones y paradojas, como generar una transformación en las mismas prácticas educativas.

Para tales fines, el trabajo será dividido en tres partes. En la primera de ellas analizaremos la propuesta Philosophy for Children de Mathew Lipman haciendo hincapié en el concepto de comunidad de indagación y el rol que se le asigna al docente en dicho programa. En la segunda parte, bajo la hipótesis de que, si bien dicha propuesta se presenta a sí misma como innovadora, subyacen ciertos elementos que permitirían poner en cuestionamiento esa calificación, intentaremos mostrar las semejanzas con ciertos elementos de lo que podría denominarse "educación tradicional"2. Para tales efectos, analizaremos comparativamente características de la propuesta de Lipman con un trabajo de Doyle (1980) sobre

1 Palabras que G. Deleuze dirige a M. Foucault en Foucault (1991).

2 Con "educación tradicional" nos referimos a concepciones educativas que se fundamentan en una relación de total asimetría entre docente y alumno/a. Este tipo de relación, que hace especial hincapié en el rol pasivo del estudiante en contraposición al rol activo del docente, fue denominada como "educación bancaria" (Freire, 2003) y enseñanza explicadora (Rancière, 2007), entre otras denominaciones. 
control de una clase. Por último, examinaremos algunos elementos del planteo ranceriano en el Maestro ignorante respecto a la relación entre docente-alumno/a. En la misma lógica de los apartados anteriores, analizaremos si efectivamente se plantea una propuesta diferente y hasta qué punto es posible pensar una relación diferente entre docentes y alumnos/as. Hecho este recorrido, pondremos en consideración aquello que creemos que debería ser el objetivo central del filosofar con niños y niñas. En suma, este trabajo se propone reflexionar sobre las prácticas docentes y sus consecuencias políticas, no con la intención de resolver las tensiones que se plantean en su seno, sino como un modo de habitarlas y convivir con ellas.

\section{Innovación y comunidad de indagación en fillosofía para niños}

Desde su aparición hacia fines de la década de 1960, el programa Philosophy for children $(\mathrm{FpN})$ se ha presentado como una propuesta de innovación educativa (Kohan, 2004, p. 110). En efecto, su objetivo era el de buscar respuestas a ciertos elementos problemáticos que Mathew Lipman, creador de este programa, encontraba en los estudiantes que llegaban al nivel universitario. Asimismo, no sólo fue presentada de ese modo por sus iniciadores, sino que igualmente ha sido recibido por quienes asistieron a sus cursos y luego expandieron la propuesta por diferentes partes del mundo.

Así, por ejemplo, observamos que Ann Sharp y Laurence Splliter, colaboradores de Mathew Lipman en el armado del programa, materiales y manuales, en su libro Teaching for Better Thinking. The Classroom Community of Inquiry, se autodefinen como críticos del sistema educativo y, sobre esa base, se proponen examinar aquellos supuestos en los que se "apoyan la mayoría de los sistemas educativos" (1996). El hecho de que la traducción del título en nuestro país haga referencia a "la otra educación" ${ }^{3}$ muestra el modo en que fue recibida esta nueva propuesta.

En esta línea, en La filosofía en el aula, leemos en la contratapa de la traducción española que, en el marco de "serios esfuerzos para mejorar los sistemas educativos", la Filosofía para niños "es una de las propuestas más completas y más sólidamente elaboradas" (1992). Uno de los puntos de partida de este libro es, en efecto, la necesidad de una transformación educativa, motivo por el cual se dedica un capítulo a rastrear aquellos puntos que es necesario transformar, en vez de modificar (1992, p. 51), para generar un verdadero cambio a nivel educacional.

3 En Argentina, el libro Teaching for Better Thinking. The Classroom Community of Inquiry fue traducido con el título, "La otra educación: filosofía para niños y la comunidad de indagación", que muestra de alguna manera el modo en que fue recibida la propuesta de Lipman en nuestro país. 
El diagnóstico que Mathew Lipman realiza para desarrollar su programa es la ineficacia del sistema escolar, reflejada en la incapacidad de estudiantes universitarios de pensar por sí mismos y argumentar de manera sólida (Echeverría, 2006). Una ineficacia que Lipman encuentra tácitamente reconocida en las continuas reformas de las prácticas educativas que se llevaban a cabo sin generar una verdadera transformación (1992, p. 51). Desde su óptica, estos enfoques que tienden a reformar sólo alcanzan a proponer educaciones compensatorias que tienden a palear los problemas existentes aunque nunca solucionarlos (1992, p. 53). De esta manera, continúa Lipman, la experiencia vivida en las escuelas se torna meramente extrínseca, en la medida que los/as niños/as se vinculan con lo que allí les ocurre de manera instrumental y carente de sentido. Se torna una experiencia vacía, sin motivación, aburrida (1992, p. 58), en gran medida porque la vida escolar se torna una rutina, en las que el conocimiento se les presenta "extraño, fragmentario y desconcertante" (1992, p. 56) para el/la niño/a.

Ahora bien: ¿de qué manera se va a producir este cambio? Uno de los conceptos centrales del programa de FpN es el de comunidad de indagación ${ }^{4}$. Si bien la propuesta inicial de Lipman ha recibido críticas y reformulaciones ${ }^{5}$, este ha sido un concepto que todos los teóricos han valorizado. López, por ejemplo, quien se aparta en relación al concepto de experiencia de la propuesta de Lipman $(2006,2008)$, reconoce que la comunidad de indagación "es la atmósfera en la que intentamos ser sensible a lo inesperado, a lo emergente" (2008, p. 91) y que su importancia se debe a que "el tiempo, la atención y el valor que damos a las palabras de los otros no tiene condiciones" (2008, p. 89). En la medida en que la comunidad de indagación es considerada el encuadre de la reflexión filosófica, observamos una línea de continuidad con Lipman, quien sostenía que el espacio en el cual el ejercicio filosófico se llevaría a la práctica sería el aula en la medida en que fuera convertida en una comunidad de indagación.

En lo que se refiere a la caracterización inicial, Lipman señala tres requisitos para que el aula se convierta en una comunidad de indagación: en primer lugar, (1) el respeto mutuo de los niños entre sí y de éstos con los profesores, (2) la ausencia de adoctrinamiento y (3) la disponibilidad hacia la razón. (1992, p. 118). En este ámbito, el docente debe "evitar abortar el pensamiento de los niños", así como tampoco "manipular la discusión para conseguir que los niños adopten las convicciones personales del profesor" pues "disentir es un derecho, no una obligación (...), el derecho a estar en desacuerdo no es mayor que el derecho a estar de acuerdo y el derecho a no estar de acuerdo" (1992, p. 119).

4 Echeverría (2006, 95, n. 23) distingue inquiry de research. Afirma que mientras que el último término hace referencia a "investigar", Lipman entiende que inquiry va más allá de investigar. Esto lo ha llevado a traducir community of inquiry como "comunidad de diálogo". Por nuestra parte, utilizaremos la traducción que comúnmente es encontrada en la bibliografía.

5 Algunos autores que han reformulado, en mayor o menor medida la propuesta de Lipman, son: Kohan $(2000,2004)$ en relación al concepto de infancia y su propuesta en término políticos, López (2006) en relación al concepto de experiencia y Santiago (2006), que, aún reconociéndose en la misma línea que Lipman, señala la necesidad de reformular las novelas filosóficas para niños/as y se distancia en relación a la utilización de manuales de acompañamiento docente. 
El punto de inflexión respecto de la propuesta tradicional, que convierte el aula en una comunidad de indagación, recae sobre el rol docente. Pues si bien debe contemplar los puntos descriptos en el párrafo anterior, a su vez debe garantizar que se respeten los procedimientos y las técnicas apropiadas que han de emplearse en el contexto de la comunidad de indagación (1992, p. 118). La relevancia del rol docente en tanto garante y condición de posibilidad para el efectivo desarrollo de la comunidad de indagación puede observarse también en la atención que ha merecido en la bibliografía especializada sobre el tema.

Lipman, por ejemplo, lo ha caracterizado como un árbitro y figura de autoridad (1992, p. 194); Splliter y Sharp, por su parte, han negado su papel como trasmisor de conocimiento (1996, p. 166), punto que Kohan y Waksman comparten, aunque señalan la necesidad de que el docentes sea autónomo respecto de las novelas y manuales para el docente creados para el programa FpN $(2009$, p. 27). Sin embargo, pese a ciertas diferencias, es posible encontrar una constante en todos ellos: la necesidad de "correr" al docente del tradicional lugar de saber (Lipman, 1992, pp. 175-176; Splitter y Sharp, 1996, pp. 186-191; Kohan, 2013, pp. 26-27; Waksman, 2000, pp. 200-203).

Retomando los tres requisitos mencionados supra nos interesa analizar la relación entre (2) y (3). Pues, ¿es la apuesta por la racionalidad (Gardella, 2014) compatible con la ausencia de adoctrinamiento? ¿Cuál es el rol del docente dentro de este encuadre? Incluso podríamos preguntarnos si es posible una práctica pedagógica carente de adoctrinamiento, pregunta que retomaremos más adelante. En la siguiente sección analizaremos si los supuestos y objetivos del programa Philosophy for children permiten lograr innovaciones. Intentaremos mostrar cómo esta apuesta por la racionalidad está estrechamente vinculada con la apuesta política que subyace a los orígenes de la Filosofía para Niños.

\section{La filosofía para niños como praxis política}

Si bien en sus inicios Lipman hizo hincapié en el desarrollo de las habilidades de pensamiento, en el pensamiento creativo y cuidadoso con el otro, lo cierto es que estos siempre fueron fines secundarios para alcanzar otro de mayor relieve. En la misma clave que el intelectualismo socrático, Lipman consideraba que la investigación pedagógica y la educación moral estaban estrechamente vinculadas (1992, pp. 285-292), y que la aplicación del programa de filosofía con niños/as en todos los niveles pre-universitarios, promovía la formación de sujetos razonables para la democracia.

Para Lipman la filosofía "educa en y para la democracia" (Kohan, 2009, p. 14) y es su adalid (Kohan, 2000, p. 19). Ante esto, podríamos preguntarnos en qué medida una propuesta de este tipo produce realmente un cambio respecto de las prácticas educativas que, como vimos en la sección anterior, el programa Philosophy for children afirmaba superar. Porque, después de todo, lo que se 
pretende con esta propuesta es formar sujetos conformes al modelo propuesto por el docente, que responde, a su vez, a los valores de ciudadanos democráticos sobre los que cimienta este programa .

En efecto, al leer con detenimiento la propuesta de Lipman observamos, por ejemplo, que uno de los motivos por los cuales las discusiones filosóficas no llegan a buen puerto es la falta de modelos de una buena discusión. Es por ello que considera "útil tener una tradición establecida de discusión que cada niño pueda asimilar automáticamente y con la cual pueda identificarse e involucrarse" (1992, p. 197).

Al respecto, consideramos que el hecho de que se hable que los/as niños/as "asimilen automáticamente" y que "puedan identificarse" es un indicio de que el docente siempre figura y posibilita una conducta ajena y externa que los/as alumnos/as deben incorporar. Si además de esto, tenemos en cuenta que el objetivo central de Filosofía para Niños es "ayudar a los niños a aprender a pensar por sí mismos" (1992, p. 129), puede observarse que un supuesto educacional es que el maestro, con la ayuda de los manuales para el docente y las novelas filosóficas, es quien ayuda al niño a aprender algo ("pensar por sí mismo") y que para que ese aprendizaje se produzca debe haber alguien que lo ayude.

A su vez, no debemos olvidar que Lipman afirma la necesidad de la racionalización del currículum, que implica que "el inmenso corpus de la filosofía, la acumulación de cientos de años de saber filosófico, sea revisado para establecer cómo puede dosificarse en los sucesivos niveles" (1992, p. 43), la necesidad de distinguir lo filosófico y lo pseudofilosófico (1992, pp. 49-50) y también compara el razonamiento con la medicina, ambas caracterizadas, una para la mente y otra para el cuerpo, como "artes curativas que intentan curar los fallos o daños a los que la mente o el cuerpo se ven sometidos" (1992, p. 130). Al preguntarnos acerca de quién produce la racionalización del currículum, quién distingue lo filosófico de lo pseudofilosófico y quién purifica las mentes, observamos que todas las respuestas conducen al maestro como garantía de esas condiciones.

Estos elementos son los que ha llevado a Kohan a caracterizar el concepto de infancia en Lipman como infancia educada (2004, p. 123), que implica, entre otras cosas, aguardar por los/as niños/as con una idea regulativa sobre cómo deberían ser. De este modo, se hace de manifiesto la íntima relación entre la filosofía para niños, como una instancia educativa más, y la política:

"Si la filosofía no llevase a los niños a ser personas democráticas o si ellos pudiesen llegar a ser así formados de otra manera, no habría necesidad de la filosofía en la escuela [según la propuesta de Lipman]. La enseñanza de la filosofía se inscribe así en una lógica de educación formadora según una ética y una política ya determinadas, anteriores, guiadoras, fundantes. La filosofía sirve al sueño democrático de Lipman" (2004, pp. 124-125). 
Estas palabras muestran que en realidad, aquella propuesta de innovación, no difiere estructuralmente de otras propuestas educativas. El docente, en tanto gestor de un ideal político, mantiene el poder y lo administra dentro del aula en aras de ese ideal. El disciplinamiento se pone de manifiesto en la medida en que las libertades que el docente ofrece a los/as niños/as están orientadas en función de los objetivos planteados anteriormente.

Porque además, ese ideal o meta que es deseable alcanzar, no es consensuado sino impuesto. Y es también regulativo, en la medida que condiciona las prácticas que se desarrollan en el marco de esta propuesta, y formadora, en la medida que se constituye como un criterio de normalidad a la que es deseable que los/ as infantes se asemejen.

Ahora bien: aunque las relaciones entre la educación y la política han sido estudiadas (Cerletti, 2008; Cullen, 2004; Ricoeur, 1984; Skliar-Tellez, 2008), dado que "la política no se muestra como un contenido curricular claro y distinto" (Berisso, 2006, p. 261), en muchos casos esos estudios buscaron señalar, como intentamos hacer nosotros anteriormente, en qué elementos subyace la dimensión disciplinatoria. Tal es el caso del Maestro ignorante de Rancière, que nos interesa analizar especialmente ya que en él, a nuestro criterio, es posible rastrear algunos elementos que pondrían en cuestión la posibilidad de pensar una práctica educativa que no se sustente sobre cierta asimetría entre docente y alumno/a.

\section{Relación docente-alumno/a en el Maestro ignorante}

La propuesta rancieriana de enseñanza universal se fundamenta principalmente en la relación que el docente establece entre su saber y el alumno. En términos prácticos Rancière propone un desplazamiento del docente del rol tradicionalmente asignado para dar lugar a la emancipación del "educando". Como señala Cerletti al respecto, Rancière "critica el que haya mediadores [maestros] que lleven a adelante la igualdad" (2003, p. 148) pues no es posible lograr igualdad partiendo de la desigualdad (2003, p. 149) ${ }^{6}$. A diferencia de toda la educación explicadora, Rancière destaca que el mérito de la educación universal propuesta por Jacotot en su experiencia en la Universidad de Lovaina, es el haber "retirado su inteligencia del juego, permitiendo que la inteligencia de sus alumnos se enfrentara con la del libro" (2007, p. 28). Esta es la condición que permite que el alumno se emancipe y verifique la igualdad de su inteligencia con la de los demás. En este marco, lo que analizaremos a continuación es si este despla-

6 Incluso Sócrates -cuyo "sólo sé que no sé nada" podría equiparase, a primera vista, con el "no tengo nada que enseñarles" (2007, p. 30) ranceriano- cometería este error. Para el pensador francés, en cada maestro explicador duerme un Sócrates, que pregunta para instruir y al interrogar, pregunta para demostrar su saber y la impotencia del aprendiz (2007, pp. 46-47). 
zamiento del maestro es genuino o si, en realidad, es también ficcional como intentamos mostrar en el caso de Lipman.

En primer lugar, debemos señalar que al narrar la experiencia de Jacotot, Rancière reconoce que "los alumnos habían aprendido sin maestro explicador pero no por eso sin maestro" (2007, p. 28). Este es para nosotros el primer indicio de que, si bien se propone un cambio en el concepto de enseñanza, se resignifica el rol del maestro aunque no se lo elimina.

Por otra parte, podríamos preguntarnos si realmente el método pertenece por completo al alumno, como sostiene Rancière (2007, p. 29), y si con este nuevo método se rompe con la asimetría que supone la educación basada en la explicación, pues resulta paradójico que el método sea del alumno cuando Rancière utiliza términos relacionados con la violencia o el forzamiento para referirse a la acción del maestro: por ejemplo, al utilizar la imagen del bosque, Rancière afirma que el maestro "da la orden de atravesar el bosque" al alumno (2007, p. 24) y que el maestro "obliga a actualizar su capacidad" (2007, p. 31).

Asimismo, esa actitud de forzamiento se ve plasmada en la verificación 7 que el maestro debe realizar respecto del alumno: "no verificará aquello que el alumno ha encontrado, sino que haya buscado. Juzgará si ha prestado atención" (2007, p. 49) ${ }^{8}$. El maestro verifica que algo se haya cumplido de acuerdo a lo propuesto. Pero esa propuesta proviene, justamente, del maestro, quien a su vez tiene la función de juzgar si el alumno ha actuado de acuerdo a esa propuesta; es decir, que se haya hecho con atención.

Incluso en el prólogo ya puede percibirse que es necesario llevar al alumno hacia el lugar que el maestro emancipador (y Rancière) considera correcto. Pues se puede "confirmar una incapacidad en el acto mismo que pretende reducirla [embrutecimiento] o, a la inversa, forzar una capacidad, que se ignora o se niega, a reconocer y a desarrollar todas las consecuencias, que se ignora o se niega [emancipación]" (2007, p. 9). En este punto parecen borrarse los límites entre la propuesta de Rancière y la enseñanza criticada en cuanto a la relación entre maestro y alumno. El "maestro ignorante" también posee un saber que el alumno debe aprender para poder emanciparse.

En último lugar, nos parece oportuno destacar que el sueño (objetivo) de este nuevo método es "que cada ignorante pudiera erigirse en el maestro de otro ignorante" (2007, p. 33). Pero "para emancipar a otro es necesario estar emancipado uno mismo. Hay que conocerse a sí mismo como viajero del espíritu, igual a todos los demás viajeros" (2007, pp. 51-52). Esto quiere decir que alguien

7 Para un análisis de las "violencias" que es posible encontrar en El maestro ignorante, véase Nózica (2012). Si bien no acordamos completamente con lo sostenido allí, consideramos que aporta elementos interesantes para analizar el libro de Rancière.

8 El caso al que remite Rancière es análogo al de la madre que verifica que se busque (2007, pp. 51). De esta manera, el auto francés muestra que, más allá del contexto, lo que se considera es quien ocupa el rol de maestro/a o guía a otro/a y cómo se vinculan ambos. 
para poder emancipar, debe estar primero él mismo emancipado. Hay un cierto "saber" previo que es necesario para que la emancipación sea posible.

Ahora bien, tomando como referencia una propuesta educativa tradicional (Doyle, 1980), hemos intentado mostrar que tanto una propuesta educativa que se presenta a sí misma como innovada (Lipman), como otra que se construye sobre la base de una fuerte crítica de las relaciones de poder entre las partes involucradas en el proceso de enseñanza-aprendizaje (Rancière), se establecen relaciones similares entre docente, alumno/a y el saber que está en juego. Surge entonces la necesidad de preguntarnos en qué medida es posible una propuesta educativa que no parta de una idea regulativa de persona, un ideal político de ciudadano, hacia la cual el/la niño/a deba ser llevado a lo largo de su vida escolar. Pues, como sostuvo Freire, "no basta con decir que la educación es un acto político (...) Es preciso asumir la politicidad de la educación” (1996, p. 52).

Por otra parte, si bien hay indicios de que Rancière de alguna manera reproduce las relaciones de desigualdad que él mismo critica, su planteamiento es interesante en la medida en que pone de manifiesto que la cuestión política en la educación se da en diferentes planos. Tal como señala Kohan al respecto, para Rancière en un acto educativo "no sólo se aprende el saber (...) sino que aprende también una relación con el saber, y sobre todo, una relación consigo mismo mediada por el 'dueño' del saber" (2009, pp. 89-90).

De este modo, teniendo en cuenta estas distinciones, el análisis del Maestro ignorante revela que hay al menos tres niveles diferentes que entran en juego en un acto educativo:

a. Enseñanza/aprendizaje de un "saber"

b. Enseñanza/aprendizaje de una relación entre quien enseña/aprende y ese "saber".

c. Enseñanza/aprendizaje del rol del que aprende en ese proceso de enseñanza/ aprendizaje.

En este sentido, Rancière remarca que ninguna práctica educativa es "inocente". En ella se ponen en juego una serie de elementos que van más allá de la enseñanza de un "saber". Porque además podríamos agregar a esta caracterización otro componente en el cual, a nuestro juicio, se plasma en mayor dimensión la cuestión política de la educación: la enseñanza y aprendizaje de la relación entre quien posee el "saber" y quien tiene que obtenerlo.

Y es precisamente sobre esta relación desigual que se fundamenta y erige la escuela como institución de control social (Foucault, 1989). De este modo, se constituye una idea de infante incompleto, que necesita de otros para poder formarse y de ponerse en relación con quien posee un saber; es decir, un infante que necesita ser educado. Esto explicaría por qué alrededor de la escuela se ha 
conformado una serie de discursos (Bourdieu, 2008) que tienden a reforzar esta relación asimétrica entre unos y otros.

¿Cuál es la noción de infancia o el lugar del niño/a en este panorama? Desde la antigua Grecia con el pensamiento platónico, el/la niño/a ha sido visto como una pieza clave en la transformación política ${ }^{9}$. Por eso se ha vuelto necesario generar mecanismos que permitan que ese ideal político, en el cual se desarrolla esa práctica educativa, se realice. En otras palabras, son destinatarios/as no sólo de "contenidos curriculares" sino también de otros "contenidos"10 que están relacionados con asimilar una manera de relacionarse con quien posee el poder en esa situación.

Por tales motivos, a nuestro criterio, el punto en cuestión es que más allá de cuál sea ese ideal político (la democracia, en el caso del programa de Lipman, o la emancipación como sostiene Rancière), hay una matriz que subyace a toda práctica educativa que es, precisamente, la de constituir subjetividades subsidiarias respecto a la figura que guía el hecho educativo. Y dado que estas relaciones, lejos de ser dinámicas y fluctuantes, tienden a ser estáticas y permanentes, en muchos casos conforman lo que Foucault (1994) denomina "estado de dominación". De esta manera, la institución escolar es propedéutica de las instituciones estatales. El paso de un individuo por aquella instancia genera las condiciones necesarias para su mejor desempeño y control en las últimas. Bajo estas condiciones, es que nos preguntábamos si es posible una práctica educativa que pueda generar rupturas respecto de estas relaciones. $\mathrm{O}$ en otras palabras, nos preguntamos:

\begin{abstract}
“(...) ¿Cómo enseñar la relación entre el poder y lo que somos, en tanto sujetos constituidos? ¿Cómo aludir a una resistencia que exprese como querría Foucault, la posible franqueabilidad de los límites impuestos a nuestra subjetividad? ¿Cómo enseñar política más allá de los marcos instituidos por el poder soberano, de las prácticas representativas de la partidocracia, de las fórmulas canónicas del parlamentarismo? ¿Cómo enseñarla a niños y jóvenes?" (Berisso, 2006, p. 262).
\end{abstract}

\title{
4. A modo de conclusión
}

A lo largo de este trabajo hemos intentado poner de manifiesto el tipo de relación entre docente-alumno/a que suele establecerse en el ámbito escolar. Lo hemos hecho a partir del análisis de diferentes propuestas que creemos representan

9 Para un análisis de la importancia que desde la antigüedad ha tenido la educación de niños y jóvenes, véase Jaeger, W. (1947) Paideia: the Ideal of Greek Culture, Blackwell.

10 En otras palabras, esto es lo que comúnmente se denomina distinción entre "contenido" y "forma". "Contenido" como aquello que se enseña, y "forma" como el modo, metodología de enseñarlo. Según nuestro análisis esta distinción se oscurece tan pronto percibimos, tras análisis como los de Rancière (2007) o Foucault (1989), que en realidad el "contenido" más importante de la educación es la "forma" misma. 
miradas diversas en torno a esa relación entre docente-alumno/a. Además, señalamos que si bien estas propuestas son diversas, todas poseen una matriz en común que consiste en mantener una relación asimétrica entre el/la infante y el/la adulto/a. Y en la medida en que toda práctica educativa es, en sí misma, una práctica política, señalamos que la función de ese tipo de relación consiste en alcanzar un modelo de ciudadano previamente instaurado.

En este sentido, y estrechamente vinculado con lo anterior, observamos que esto implica, por un lado, partir de una concepción de infante como carencia o incompletitud, pues se concibe la función docente como aquel guía y mentor para la realización de un tipo determinado de ciudadano. Por otro lado, dada esa premisa de niño/a como ser ontológicamente incompleto/a, esto también implica recibir con ciertas expectativas vinculadas con un ideal de ciudadano que condiciona directamente la práctica educativa en todas sus dimensiones.

En segundo lugar, hemos intentado remarcar que es complejo (sino imposible) pensar una práctica educativa y política en la que no subyaga un ideal político de ciudadano. Esa es una de las condiciones fundantes de las instituciones escolares. Incluso, yendo aún más allá de los límites de lo escolar, consideramos que en todas las esferas en las cuales se establece algún tipo de relación entre personas, se ponen en juego aquellos ideales de cada uno. Pues, como sostuvo Sartre en el Existencialismo es un humanismo,
“Cuando decimos que el hombre es responsable de sí mismo, no queremos decir que el hombre es responsable de su estricta indi- vidualidad (...) En efecto, no hay ninguno de nuestros actos que al crear al hombre que queremos ser, no cree al mismo tiempo una imagen del hombre tal como consideramos que debe ser. Elegir ser esto o aquello, es afirmar al mismo tiempo el valor de lo que elegimos" (Sartre, 1999, p. 15).

En otras palabras, siempre estamos proyectando un ideal subjetivo hacia el mundo con cada palabra y acto que realizamos. Y las prácticas educativas, por supuesto, no escapan a esta lógica. En este sentido, es que no preguntamos qué tan posible es plantear una propuesta educativa de filosofía con niños y niñas que escape a la matriz mencionada supra. ¿Es posible pensar una educación emancipadora de la propia educación? ¿En qué sentido sería posible?

En primer lugar, afirmamos que es posible. En segundo, que es precisamente la filosofía a quien corresponde llevarla a cabo. Pues, “¿qué es la filosofía hoy -quiero decir la actividad filosófica- si no el trabajo crítico del pensamiento sobre sí mismo?" (Foucault, 1998, p. 11). El trabajo crítico, en este caso, debe centrarse en las propias prácticas educativas. Debe ser un cuestionamiento a las prácticas educativas desde las mismas prácticas educativas. Es necesario poner en cuestión aquellas prácticas habituales de control y jerarquización, así como también revisar las propias conductas e interpretar los signos de resistencia que nos trasmiten niños y niñas en las escuelas. 
No obstante esto, no podemos dejar de mencionar que lo paradójico de esto es que una propuesta realmente liberadora debe conducir a su propia desaparición. En efecto, al tratarse de una propuesta que, al fin al cabo, pretende poner en cuestionamiento el punto de partida de la misma propuesta, se convierte en una práctica auto-denunciatoria en la medida que llevarla a cabo conlleva su propia finalización.

En esto consiste, haciéndonos eco del acápite de nuestro trabajo, el "dar voz" a quienes en un principio no la tienen, pero una voz que se vuelva contra aquello que posibilitó su propia pronunciación. Consiste en recibir lo nuevo, en términos de Kohan, sin más. Pero no algo "nuevo" en el sentido de diverso, porque en tal caso podríamos caer en la tentación de subsumir esa novedad en lo que ya estaba, valorándolo en función de aquello que posibilitó su aparición. No. Lo que se trata es de recibir a lo "nuevo", incluso aquello "nuevo" que reemplace, desplace o finalice lo que venía siendo, como una nueva posibilidad de fundar una nueva educación que se sustente, no en la asimetría entre docentealumno/a sino en una educación que apueste a la igualdad de los diferentes, la no jerarquía, la no representatividad (Kohan, 2004, p. 279). En este sentido, la filosofía con niños y niñas se erige como un espacio para propiciar una nueva práctica educativa. Una práctica en la que nadie, ni docentes ni alumnos/as, esté habilitado a pensar, ni hablar por nadie. Una práctica desconocida hasta el momento de concretarse.

\section{Referencias}

Agamben, G. (2007 [1978]). Infancia e historia. Destrucción de la experiencia y origen de la historia. Buenos Aires: Adriana Hidalgo.

Berisso, D. (2006). La educación política en el marco de la educación como política. En Kohan, W. (Comp.). (2006) Teoría y práctica con niños y jóvenes: experimentar el pensar, pensar la experiencia. Buenos Aires: Noveduc, pp. 261-266.

Caputo, C. (2006). Algunas consideraciones acerca de la filosofía como invitación a pensar. En Kohan, W. O. (Comp.). Teoría y práctica con niños y jóvenes: experimentar el pensar, pensar la experiencia. Buenos Aires: Noveduc, pp. 33-38.

Cerletti, A. (2008). Repetición, novedad y sujeto en la educación. Un enfoque filosófico y político (La educación como problema filosófico y político, pp. 13-24, y El sujeto educativo y el sujeto de la educación, pp. 93-120). Buenos Aires: Del estante.

Cerletti, A. (2003, mayo-agosto). La política del maestro ignorante: la lección de Rancière. Revista de Educación y Pedagogía, XV (36), 145-151. 
Cullen, C. (2004). La educación ética entre el disciplinamiento social y la madurez individual de los sujetos". En C. Cullen, Perfiles ético-políticos de la educación (pp. 109-123). Buenos Aires: Paidós.

Derrida, J. (1990). Du droit à la philosophie. París : Galilée, 1990.

Echeverría, E. (2006). Filosofía para niños. México D.F: Sm de Ediciones.

Ferraro, G. (2013). Repensando, con otras voces, los sentidos del filosofar". En Kohan W. O., \& B. F. Olarieta (Coords.). La escuela pública apuesta al pensamiento. (pp. 165-234). Rosario: Homo Sapiens.

Foucault, M. (1989). Vigilar y castigar: nacimiento de la prisión. Madrid: Siglo XXI España Ediciones.

Foucault, M. (1994). La hermenéutica del sujeto. Madrid: Ediciones de La Piqueta.

Gardella, M. (2014). Coloquio Internacional (FFyL, UBA) XXI Jornadas sobre la Enseñanza de la Filosofía: El valor del sinsentido en la práctica de filosofar con niños y niñas. Inédito.

Kant, I (1943). Crítica de la razón pura (T.II). Buenos Aires: Losada.

Kohan W., \& Olarieta, B. F. (Coords.). (2013). La escuela pública apuesta al pensamiento. Rosario: Homo Sapiens.

Kohan, W. (1996). Filosofía para niños: elementos para pensar una idea. Versiones, vol. 6, 58-65.

Kohan, W. (2000). Fundamentos de una filosofía en el aula. En Kohan, O. W., \& Waskman, V. (comp.). La filosfía para niños. Discusiones y propuestas (pp. 191-202). Buenos Aires: Noveduc..

Kohan, W. (2004). Infancia entre filosofía y educación. Barcelona: Laertes.

Kohan, W. (2007). Infancia, política y pensamiento. Buenos Aires: Del estante.

Kohan, W. (2013). Palabras, pasos y nombres para un proyecto. En Kohan W. O., \& B. F. Olarieta (Coords.). La escuela pública apuesta al pensamiento (pp. 15-50). Rosario: Homo Sapiens.

Kohan, W. (2013). El maestro inventor. Simón Rodríguez. Buenos Aires: Miño y Dávila Editores.

Kohan, W. (Comp.). (2006). Teoría y práctica con niños y jóvenes: experimentar el pensar, pensar la experiencia. Buenos Aires: Noveduc. 
Lipman, M. (1988). Philosophy Goes to School. Philadelphia: Temple University Press.

Lipman, M. (1991). Thinking in Education. Cambridge: University Press.

Lipman, M., Sharp, A., \& Oscanyan, F. (1992). La filosofía en el aula. Madrid: Ediciones de la Torre.

López, M. (2006). 'Filosofía con niños': crónica de una feliz confusión en torno del concepto de experiencia. En Kohan, W. (Comp.). (2006). Teoría y práctica con niños y jóvenes: experimentar el pensar, pensar la experiencia (pp. 25-38). Buenos Aires: Noveduc.

López, M. (2008). Filosofía con niños y jóvenes. La comunidad de indagación a partir de los conceptos de acontecimiento y experiencia trágica. Buenos Aires: Centro de publicaciones educativas y material didáctico.

Merleau-Ponty, M. (1993 [1945]). Fenomenología de la percepción. Barcelona: Planeta- De Agostini.

Nózica, G. (2012). Las violencias del maestro ignorante de Rancière. Revista sobre enseñanza del Derecho, 10(19). 273-288.

Rancière, J. (2007 [1987]). El maestro ignorante: cinco lecciones sobre la emancipación intelectual. Buenos Aires: Del Zorzal.

Santiago, G. (2006). Filosofía, niños, escuela: trabajar por un encuentro intenso. Buenos Aire: Paidós.

Skliar, C. (2011). Lo dicho, lo escrito, lo ignorado. Ensayos mínimos entre educación, filosofía y literatura. Buenos Aires: Miño y Dávila.

Splitter, L., \& A. Sharp (1996). La otra educación. Buenos Aires: Ediciones Manantial.

Waskman, V. (2000). ¿Quién es el maestro de filosofía? En Kohan, O. W., \& Waskman, V. (comp.). La filosofía para niños. Discusiones y propuestas (pp. 191-202). Buenos Aires: Noveduc. 